O. Tajima ${ }^{1,3}$. H. Nguyen $^{2}$. C. Bischoff ${ }^{3,4}$.

A. Brizius ${ }^{3}$. I. Buder ${ }^{3}$. A. Kusaka ${ }^{3}$

\title{
Novel calibration system with sparse wires for CMB polarization receivers
}

08.01.2011

Keywords CMB, polarization, calibration

Abstract $B$-modes in the cosmic microwave background (CMB) polarization is a smoking gun signature of the inflationary universe. To achieve better sensitivity to this faint signal, $\mathrm{CMB}$ polarization experiments aim to maximize the number of detector elements, resulting in a large focal plane receiver. Detector calibration of the polarization response becomes essential. It is extremely useful to be able to calibrate "simultaneously" all detectors on the large focal plane. We developed a novel calibration system that rotates a large "sparse" grid of metal wires, in front of and fully covering the field of view of the focal plane receiver. Polarized radiation is created via the reflection of ambient temperature from the wire surface. Since the detector has a finite beam size, the observed signal is smeared according to the beam property. The resulting smeared polarized radiation has a reasonable intensity (a few Kelvin or less) compared to the sky temperature ( $\sim 10 \mathrm{~K}$ observing condition). The system played a successful role for receiver calibration of QUIET, a CMB polarization experiment located in the Atacama desert in Chile. The successful performance revealed that this system is applicable to other experiments based on different technologies, e.g. TES bolometers.

PACS numbers: 98.80.Es, 07.57.Hm, 95.85.Bh

\section{Introduction}

The primary goal of cosmic microwave background (CMB) polarization experiments in the next decade is to detect the degree-scale $B$-modes (curl components).

1:High Energy Accelerator Research Organization (KEK), 1-1 Oho, Tsukuba, Ibaraki 305-0801, Japan; E-mail: osamu.tajima @kek.jp

2: Fermi National Accelerator Laboratory, Batavia, IL 60510, USA

3: Kavli Institute for Cosmological Physics, Department of Physics, Enrico Fermi Institute, The University of Chicago, Chicago, IL 60637, USA

4: Current address: Harvard-Smithsonian Center for Astrophysics, 60 Garden Street MS 43, Cambridge, MA 02138, USA 
In the generic theories of inflation, they are induced by primordial gravitational waves. Therefore, the detection of $B$-modes is a smoking-gun signature of inflation ${ }^{1}$. The $B$-modes intensity is represented by the tensor $(T)$-to-scalar $(S)$ ratio $r \equiv T / S$. The major models predict the range of $r=0.01 \sim 0.1$.

Since the single detector sensitivity is practically limited by finite photon statistics of the CMB itself as well as noise from atmospheric emission, the generic strategy of upcoming CMB polarization experiments is better sensitivity via maximizing the number of detectors. It results in having a large focal plane receiver. While experiments have used astrophysical point sources to calibrate detectors one-at-a-time, this strategy is impractical for large channel counts. In addition to the ephemeris limitation of the point sources, they are also typically weak $(\sim \mathrm{mK}$ level) and so long exposures are needed. Therefore, it is essential to have a technique that rapidly calibrates many detectors simultaneously.

Two calibration parameters are important to minimize systematic bias: relative responsitivities and relative detector angles (orientations of the polarization responses). Especially for antenna-coupled bolometers, relative responsivities are amongst the most important calibration parameters. Polarization response is described with two Stokes parameters $Q$ and $U$. A pair of antennas, orthogonal to each other, pick up the signal components for each direction, and $Q$ (or $U$ ) is the difference between the powers seen in each direction. Therefore, imbalance of relative responsivities between a pair of bolometers causes a fake polarization. The bias in the $B$-modes power is mainly caused by the combination of the fake polarization and the (unpolarized) CMB temperature anisotropy. Calibration of the relative angles is also important for minimizing the bias due to the $E$-mode mimicking a $B$-mode.

Knowledge of the relative responsivities to $0.3 \%$, or the relative angles to $0.5^{\circ}$, would give an error on $r$ of 0.01 . To meet these requirements, QUIET, a CMB polarization experiment based in the Atacama desert plateau in Chile, utilized a large sparse wire grid calibration system.

\section{Calibration System}

The overview of the QUIET calibration system is shown in Fig. 1 and 2. QUIET's calibration system was used to study both $43 \mathrm{GHz}$ and $95 \mathrm{GHz}$ polarization detectors $^{2,3}$. In this paper, we present its performance in the later case. Parallel metal wires (wire grid) are fixed on a glass epoxy ring ( $\phi 57 \mathrm{~cm}$ of inner diameter) with $12.7 \mathrm{~mm}$ pitch and 25 grams of tension. The wire consists with $25 \mu \mathrm{m}$ diameter tungsten and coated with $0.1 \mu \mathrm{m}$ of gold. The glass epoxy ring containing the wires, is mounted on a steel ring, which itself is held next to the QUIET receiver window via three wheel bearings. A motor is used to rotate the steel ring. The wire grid size is large so that it fully covers each detector's beam envelope. Each detector sees $\approx 8$ wires in its field of view, and none of the other mechanical components. The distance between the cryostat window and wires is approximately $5.7 \mathrm{~cm}$, and was as small as possible to minimize the interference with QUIET's telescope optics.

The mechanism to generate the polarization signal is quite simple. The ambient temperature $(\sim 300 \mathrm{~K})$ is reflected from the surface of the wire, and then enters into the receiver focal plane. Since the electric field of the reflected radiation 


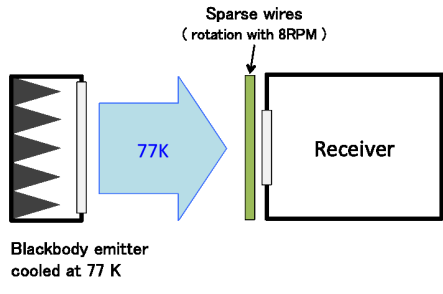

(a) System setup in the laboratory

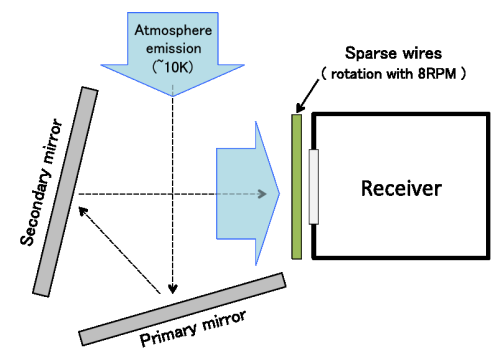

(b) System setup on the telescope mount

Fig. 1 (Color online) Overview of the system setup in the (a) laboratory, and (b) on the telescope mount at the observation site (in case of the QUIET experiment ${ }^{2}$ ). Not shown in (b) is the ground shield covering the entire telescope mirror and receiver. The sparse wire grid rotates in front of the receiver. Reflection of ambient temperature $(\sim 300 \mathrm{~K})$ from the surface of the wires creates a polarized signal, which is seen by detectors in the receiver. The magnitude of the polarized signal is effectively a few Kelvins.

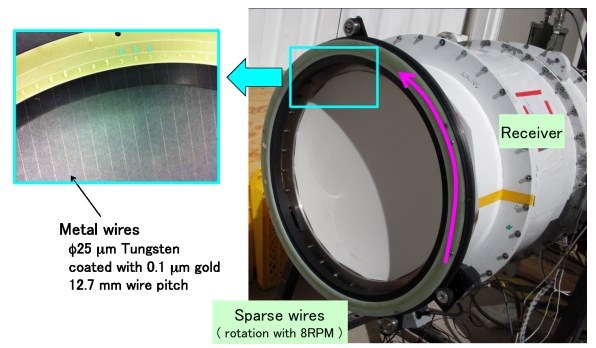

Fig. 2 (Color online) Sparse wires on a glass epoxy ring with $12.7 \mathrm{~mm}$ pitch. The ring is mounted in front of the window of the receiver, and the ring as well as the wires are rotated.

is aligned with the wire direction, reflected radiation is polarized. Each detector has finite beam resolution, therefore, the detected polarization is smeared with the beam profile; detected polarization signal is proportional to the products of the inverse of the wire pitch, beam profile and the difference between the reflected ambient temperature and the background load temperature. We obtained $\approx 2 \mathrm{~K}$ $(\approx 1.6 \mathrm{~K})$ of the polarization signal with $10 \mathrm{~K}(77 \mathrm{~K})$ load in this configuration.

By rotating the steel ring carrying the wires, it is possible to vary the angle between the detector angle $(\gamma)$ and the direction of the polarization, i.e. the direction of the wires $(\theta)$. A sinusoidal response is observed by the polarization detector as a function of the rotation angle,

$$
S(\theta)=G \cdot P \cos (2(\theta-\gamma)),
$$

where $S$ is the detector response, $G$ is the detector responsivity, and $P$ is the intensity of the polarization signal. Note that one cycle of the sinusoidal response is twice the rotation cycle. From the amplitude and phase of the sinusoidal response, we can extract simultaneously the responsivity and the detector angle. Since the wire density is uniform and the detectors have identical beam profiles, each detector observes the same signal magnitude. Moreover, the direction of the input 
polarizations is perfectly aligned, as all wires are fixed in parallel $\left(\ll 0.1^{\circ}\right.$ precision). Therefore, the system allows us to calibrate simultaneously all detectors in a short amount of time ( e.g. $30 \mathrm{sec} \sim$ a few minutes).

\section{Performance Tests}

Tests in the laboratory We performed system tests in the laboratory with the set up as shown in Fig. 1-(a). The blackbody emitter ${ }^{4}$ was housed in a vessel containing liquid nitrogen, and has a transparent window ${ }^{5}$ allowing the $77 \mathrm{~K}$ black body radiation to illuminate the detector vessel. With a constant rotational speed (rotation frequency $f$ ), the rotation angle $\theta$ is proportional to time $(t)$, i.e. $\theta \propto 2 \pi f t$. We observed a clear sinusoidal response as a function of the wire rotation $(\sim 8 \mathrm{RPM})$ as shown in Fig. 3. The response is well matched to the expectation of Eq. (1). By using only $30 \mathrm{sec}$ of data, we measured the relative angles and relative responsivities to $0.3^{\circ}$ and $1 \%$ statistical precision respectively. The detector responsitivity has been characterized via another method using a rotating metallic mirror ${ }^{6}$. This method confirms the $1.6 \mathrm{~K}$ polarization signal from the wires. In practice, we use the system to acquire the relative calibrations amongst the detectors. So it is not necessary to know the magnitude of the polarization signal precisely. Nevertheless, it is worthwhile to know the signal intensity, as it may be useful for different configurations and for other experiments.

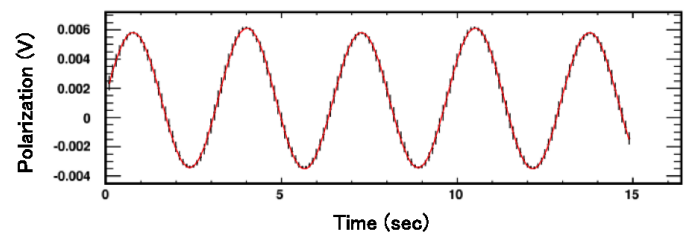

Fig. 3 Polarization response as a function of time with the wire rotation $(\sim 8 R P M)$ in the laboratory. A sinusoidal response is clearly observed with a period of twice the rotation frequency.

Tests at the observation site Field tests were performed at the QUIET observation site (Atacama desert in Chile, 5,080 $\mathrm{m}$ above the sea level), using the setup configuration shown in Fig. 1-(b). We also observed the sinusoidal response curves in all detectors as shown in Fig. 4. This is the good demonstration that we can calibrate the relative responses among detectors simultaneously by using this calibration system.

In closer examination of the measured curves, the detector responses did not follow perfectly the sinusoidal curves. The periodical distortions, or asymmetries in the response curves, were observed, which were not seen in the laboratory setup. The distortions were caused by a non-uniformity in the reflected radiation, as evident in Fig. 1-(b). The radiation from the sky $(\sim 10 \mathrm{~K})$ is much different from the ambient temperature $(\sim 300 \mathrm{~K})$. The sky temperature as well as the ambient temperature are reflected from the wires. However the relative amount depends on the 


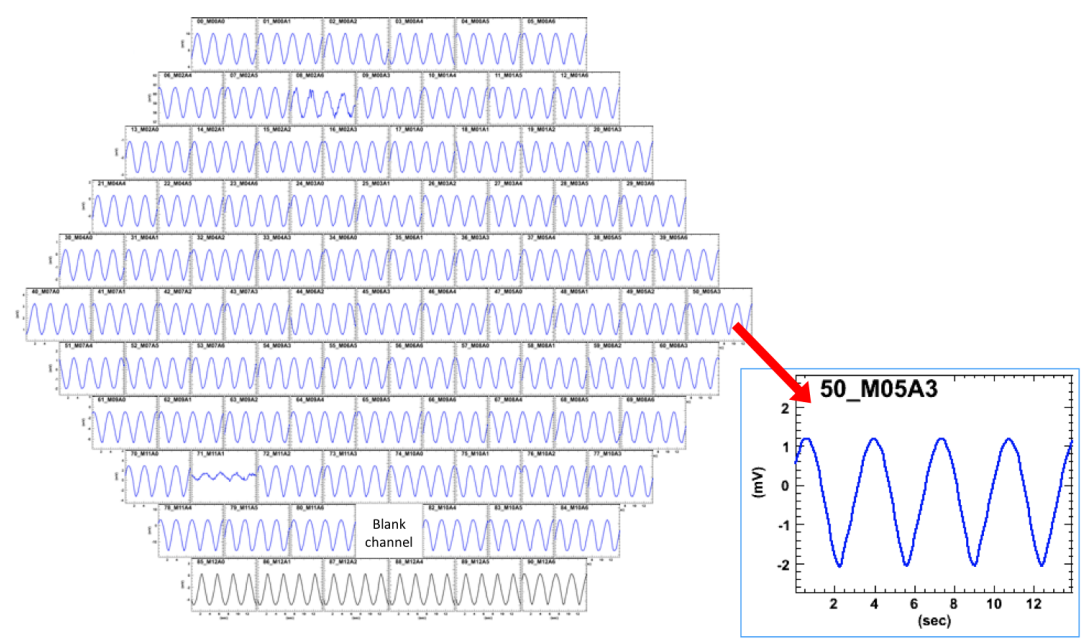

Fig. 4 Polarization responses at the observation site. We observed simultaneous sinusoidal responses for all 90 detectors in the large focal plane $(\phi \sim 30 \mathrm{~cm})$, varying with the rotation of the wires.

wire direction. The fraction of ambient temperature radiation is maximized (minimized) when the wire direction is parallel (perpendicular) to the telescope boresight axis. The QUIET detectors can measure both polarization and total power. In the case of a uniform reflection intensity, the total power response should not depend on the wire rotation angle. However this is in contrast to our situation, where a total power response variation is clearly seen (see Fig. 5).

Simulations assuming a nonuniform reflected radiation predicts: a) the total power response varies periodically with every half rotation of wires, and b) makes periodical distortions on the polarization response. We confirmed similar periodical variations in the data. Such a systematic bias can be resolved by fitting for the polarization response $(S(\theta))$, using the observed total power response $(I(\theta))$, and floating a constant offset $\left(I_{0}\right)$,

$$
S(\theta)=G \cdot\left(I(\theta)-I_{0}\right) \cos (2(\theta-\gamma)) .
$$

The precision of calibration is limited by the stability of the total power response, which is generally much worse than that of the polarization response. Thus far, the precision of the calibration is limited to $0.8^{\circ}$ and $2.8 \%$ for relative angles and relative responsivities, respectively. By orienting the wire ring in a manner to take advantage of rotational symmetries in the telescope and ground shield housing, we can, in the future, eliminate this bias. Therefore, for the future, we have prospects that the in-field calibrations can be improved to the same level as the in-lab calibrations.

\section{Conclusions}

In the goal of CMB $B$-mode detection, which is the smoking gun signature of inflation, the calibration of the relative angles and relative responsivities amongst 

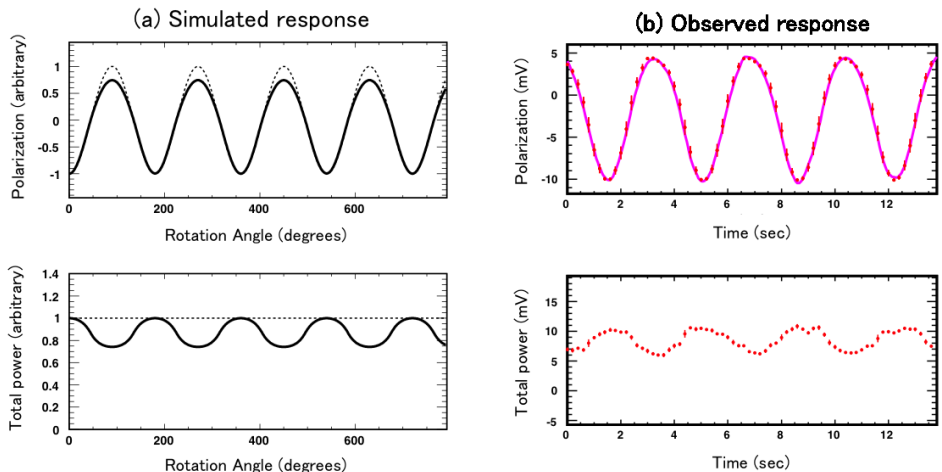

Fig. 5 Simulated responses (a) for polarization (top panel) and for total power (bottom panel). In the case where the reflected radiation is uniform (dashed lines), the total power response should be constant. On the other hand, non-uniformity in the reflected radiation makes periodical distortions on the total power response as well as on the polarization response (solid lines). In the simulation, we modeled assuming that the radiation from the sky ( $\sim 10 \mathrm{~K})$ is significantly smaller than the ambient temperature ( $\sim 300 \mathrm{~K})$. Panel (b) shows the effect as seen in the data. The solid line in the top panel is the result of the fit to Eq. (2).

the detectors becomes quite important. One needs a system to calibrate simultaneously all detectors in a given focal plane. We developed a novel calibration system that rotates a sparse metal wire grid in front of the QUIET receiver. The grid fully covers the field of view of the focal plane. The polarized radiation is created via reflection of the ambient temperature from the wire surface. The principle and performance of the system were confirmed by using the QUIET receiver. Thus far the calibration precision is systematically limited by non-uniformity of the reflected radiation. However we have prospects for improving the reflection uniformity, by choosing an appropriate configuration. Though built for QUIET, the successful performance indicates that this system is applicable for other experiments based on different technologies, e.g. TES bolometers.

Acknowledgements We acknowledge support from JSPS with Grant-in-Aid for Scientific Research on Young Scientists (A) 23684017 and (B) 20740158. We thank John Korienek, Carl Lindenmeyer, and Wanda Newby of Fermilab's Particle Physics Division, for leadership and contribution to the mechanical design and construction of the wiregrid system.

\section{References}

1. M. Zaldarriaga and U. Seljak, Phys. Rev. D 55, 1830-1840, (1997).

2. QUIET collaboration, arXiv:1012.3191 [astro-ph.CO] (2010).

3. K. A. Cleary, Proc. SPIE, 7741, 77412N (2010).

4. ECCOSORB CV-3 from Emerson \& Cuming Microwave Products, Inc., http://www.eccosorb.com/america/english/page/63/eccosorb .

5. Zotefoam PPA-30 from Zotefoam Inc., http://www.zotefoams.com/.

6. Its principle is summarized in M. Hasegawa, O. Tajima et al., Rev. Sci. Instrum. 82, 054501 (2011), see also references. 